\title{
||||||||||||||||||||||||||||||||||||||||||||||||||||||||||||||||||.
}

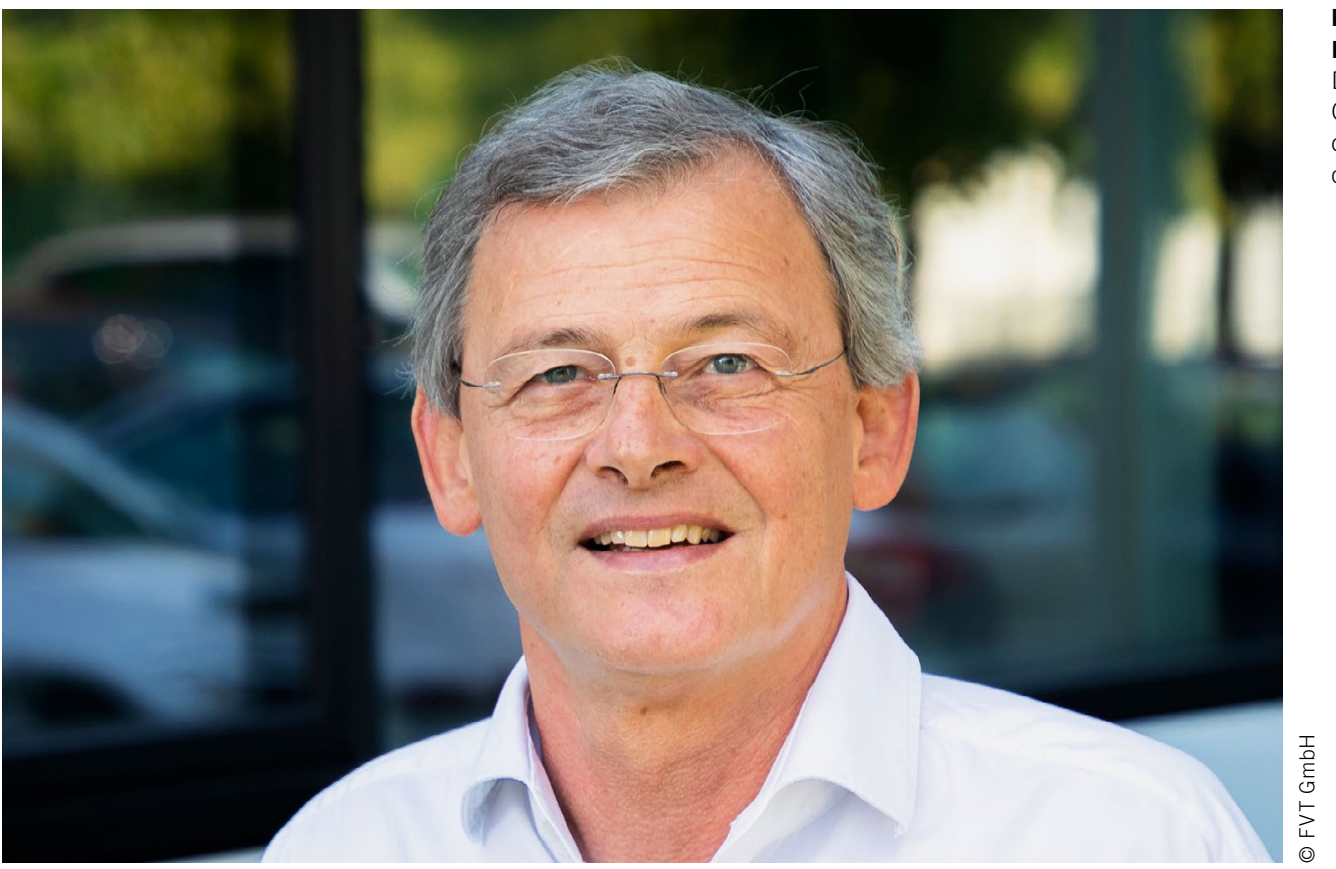

Prof. Dipl.-Ing.

Dr. Helmut Eichlseder

Director of the Institute of Internal

Combustion Engines and Thermo-

dynamics at Graz University

of Technology (Austria)

\section{Defeat Devices in Cars}

Many legal cases concerning the issue of so-called defeat devices that act on mechanisms to reduce emissions from cars are still underway. The existence of these devices was first revealed during the diesel scandal and they were initially intended to shut down or reduce the impact of systems for exhaust gas recirculation or for dispensing SCR additives which aimed to lower emission levels. They were not designed to combat one specific emission-reduction technology. Their primary purpose was to detect an emission measurement process taking place (cycle beating) and to reduce the use of these technologies or completely disable them, with the result that emission levels were much higher in real-life operation. In this case it is relatively easy to identify that these measures are illegal and even fraudulent. The fact that a defeat device of this kind, which includes cycle beating, is against the law is no longer under discussion, because this was established by the German Federal Motor Transport Authority (KBA) as long ago as 2015 and does not need to be considered further here.

Over and above this, several lawsuits have recently started with the aim of determining the legality of temperature-dependent emission reduction measures such as exhaust gas recirculation based on the ambient temperature, also known as thermal windows. In my view and from the perspective of an engineer with no formal legal training, this situation is more difficult to judge. I am not intending to gloss over or trivialize the matter, but it is in the very nature of technical systems to be subject to restrictions and limits caused by thermodynamic effects such as condensation processes, general material properties and thermomechanical stresses. The starting performance, response characteristics and behavior under full load of a vehicle cannot be expected to remain the same across the entire potential temperature range of -25 to $+40{ }^{\circ} \mathrm{C}$ or at different altitudes (and therefore air pressures). In the case of electric vehicles for example, variations in these conditions lead to significant differences in the range.

We can only hope that the legal judgments on the functionality of the emission reduction measures focus primarily on maintaining air quality and on the real benefits for the environment. In the same way as with a statistical tolerance calculation, it is important that the maximum combination of all the negative parameters that is theoretically possible is not allowed to determine the design (which would in this case anyway be very difficult to create). Instead its relevance for air quality must be the central consideration. The introduction of the RDE legislation is a step in this direction which has justifiably been described as significant and the regulations already include representative specifications of the environmental conditions.

In contrast to a design that is solely focused on extreme conditions and requires an unrealistic use of resources, a solution which aims to improve air quality will soon become widely used and therefore have a genuine impact. We must hope that this factual assessment will be reflected in the forthcoming legal cases. 